Original Research Article

\title{
Correlation between ocular axial length, anterior chamber depth, Average K-values and a differential analysis in short
}

\author{
Savitha DP. ${ }^{1}$, Sangapur AN. ${ }^{2}$, Lakshmi BR. ${ }^{3}$ \\ ${ }^{1}$ Dr. Savitha D P., ${ }^{2}$ Dr. Anusha N. Sangapur, ${ }^{3}$ Dr. Lakshmi B R. All author are Senior Residents, Department of Ophthalmology, \\ KIMS Hubli, Karnataka, India.
}

Corresponding Author: Dr. Anusha N. Sangapur, Department of Ophthalmology, KIMS Hubli, Karnataka, India. Email: anusha90ravi@gmail.com

\begin{abstract}
Introduction: To study correlation between ocular axial length, Average K-values and anterior chamber depth in eyes and to differentially analyse strength of this correlation in eyes with short axial length. Materials and Methods: The present study conducted an observational study where keratometry and ultrasound biometry records of 100 patients reporting for cataract surgery were taken. A comparative analysis using ultrasound Biometry and automated keratometry for pre-operative measurement of Axial length (AL), Average K-value, Anterior chamber depth (ACD) and calculated IOL power was done. Statistical Analysis was done to determine overall correlation between three parameters. Patients were then classified into various groups of short AL for a differential comparison within the group. Kendall-Pearson correlation test was used to assess relationship between AL and ACD, overall and within each subgroup. A P -value $<0.05$ was considered as statistically significant. Results: Comparison of axial length with various clinical parameters showed a p- value of 0.00 , suggestive of highly significant statistical correlation of ACD between 2 sub-groups (Group A; 20.00 to $20.99 \mathrm{~mm}$ and Group B; 21.00 to 22.00) and Correlation analysis between AXL, ACD and Average K showed p -value of 0.00 , suggestive of highly significant statistical correlation between AXL and ACD. Conclusions: As mean AL decreases ACD also decreases. In eyes with short AL there is wide variability in ACD which is either due to variable lens thickness or lens location in different eyes.
\end{abstract}

Keywords: Anterior chamber depth, Axial length, Average k-value, Intraocular lenses, Short eyes

\section{Introduction}

Cataract affects nearly 9-12 million Indians annually after the age of 50 years [1] And, this exceeds glaucoma, macular degeneration, and diabetic retinopathy numbers combined. Cataract surgery is the most frequently performed surgery in India, with a success rate of nearly $98 \%$ and excellent visual rehabilitation [1]. More than a million intraocular lenses (IOL) are implanted every year [2]. Cataract surgery has achieved a degree of precision where it has become a keratorefractive procedure and an expectation of complete visual rehabilitation in terms of visual acuity for distance and near, contrast sensitivity and depth of focus.

The most common reason for poor visual result in spite of an impeccable surgery is miscalculation of IOL power which makes it the most crucial component of the presurgical workup. The evolution of IOL formulae can be classified on the basis of their derivation as (a) theoretical formulae, (b) formulae based on regression analysis, and (c) a combination of both principles. Various phases of IOL

Manuscript received: $6^{\text {th }}$ November 2019

Reviewed: $16^{\text {th }}$ November 2019

Author Corrected: $24^{\text {th }}$ November 2019

Accepted for Publication: $28^{\text {th }}$ November 2019 power Formulae evolution have been labeled as various generations. Initially, a power estimation method was used, and the patient's preoperative refraction was taken into account. Later, this evolved into a more specific calculation based on biometric parameters. First-generation formulae, such as SRK was based on the regression to estimate power of the lens which was based on cornea $(\mathrm{K})$ and the Axial length (AL), Along with A-Constant of the specific IOL, to estimate power for IOL.

They were found to be accurate in average sized eyes, but for shorter or longer eyes these formulae were not reliable. In second generation formulae, a modification was made in SRK-II [3]. The third generation entailed moving from regression-based formulae to theoretical formulae that helped increase accuracy further by using biometric data to estimate the postoperative effective lens position (ELP) within the eye. The commonly used third-generation formulae were Holladay 1, the SRK-T, and the Hoffer Q. Each of these formulae estimates ELP based on $\mathrm{K}$ reading and/or axial length (AL) and the results have been more accurate results with simplicity and utilize only two 


\section{Original Research Article}

biometric data points, i.e., keratometry (K) and AL [4]. Latest fourth-generation formulae use additional biometric parameters and critically depend on ELP. but this is indeed the only parameter that cannot be measured accurately preoperatively.

ELP is defined as the effective distance between the anterior surface of the cornea and the lens plane if the lens was infinitely thin. ELP is the main limiting factor for refractive predictability after cataract surgery, as the accuracy of AL and $\mathrm{K}$ reading has now been established [5].

In spite of all these refinement refractive surprises still occur, and therefore, predictability of ELP based on AL and anterior chamber depth (ACD) were revisited. The present study observed 100 eyes and determined the correlation between AL and ACD.

The intuitive view is that with increasing average AL average ACD should also increase proportionately. It was also observed the same trend.

However, a differential analysis of AL and ACD in the eyes of short AL revealed marked variation in ACD. This prompted us to evaluate if there was indeed a correlation between AL and ACD. This study measured the degree of correlation between overall AL, Avg k-value and ACD in eyes undergoing Cataract surgery.

\section{Materials and Methods}

Sample size- 100 eyes from 100 patients

Source of data- patients attending outpatient department of Ophthalmology for cataract surgery

Duration of study- November 2015 to August 2017

Type of study- prospective correlation analysis

Sampling method - Random table number

Ethical clearance - The study protocol was approved by the Ethics Committee.

Inclusion criteria includes

1. Axial length $(\mathrm{AL})<22.00 \mathrm{~mm}$.

2. Presence of cataract.

\section{Results}

A total of 100 eyes were studied together and then allocated to two groups based on AL. In the current study, 9 patients had pre-senile cataract and remaining 91 patients has senile cataract. The study included 39 males and 61 females. The age of the patients ranged from 34 to 88 years, with mean age being $62.59 \pm 11.29$ years.

Overall, for 100 patients together mean AL was 21.36 $\pm 0.5 \mathrm{~mm}$ (range 19.98-21.99 mm), mean ACD was $3.38 \pm 0.52 \mathrm{~mm}$ (range 2.28-4.22 mm) and there was a weak positive correlation of 0.13 which was statistically significant $(\mathrm{P}<0.000)$. As the overall mean AL increased, overall mean ACD also increases.

Tropical Journal of Ophthalmology and Otolaryngology

Available online at: www.medresearch.in

453|P a g e 


\section{Original Research Article}

Group 1 had eyes with AL 20.00-20.99 mm were considered $(\mathrm{n}=23)$. Mean AL was 20.52mm and mean ACD was $2.65 \pm 0.24$ $\mathrm{mm}$ having a positive correlation of 0.86 and $\mathrm{P}=0.00$.

Group 2 had eyes with AL 21.00-22.00 mm $(\mathrm{n}=77)$ with mean AL of $21.65 \mathrm{~mm}$ and mean ACD was $3.60 \pm 0.36 \mathrm{~mm}$ with a positive correlation of 0.14 with $\mathrm{P}=0.03$

It is seen that while the correlation between overall AL and ACD was significant, there was no significant correlation with other parameters

Table-1: comparison of various parameters with the axial length.

\begin{tabular}{|l|c|c|c|}
\hline AXL $(\mathbf{m m})$ & No. of eyes & Average K & ACD \\
\hline $20.00-20.99$ & 23 & $43.71 \pm 2.55$ & $2.65 \pm 0.24$ \\
\hline $21.00-22.00$ & 77 & $44.54 \pm 2.08$ & $3.60 \pm 0.36$ \\
\hline t value & & 1.42 & $0.00 * *$ \\
\hline P value & & $0.17, \mathrm{NS}$ & \\
\hline Unpaired t test & & & \\
\hline $\mathrm{P}>0.05, \mathrm{NS}$ & & & \\
\hline
\end{tabular}

Comparison of axial length with various clinical parameters showed a p- value of 0.00 , suggestive of highly significant statistical correlation of ACD between 2 sub-groups

Table-2: Descriptive information on various preoperative clinical parameters (Overall)

\begin{tabular}{|c|c|c|}
\hline Parameter & Mean \pm SD & Range \\
\hline K1 (D) & $44.52 \pm 2.18$ & $38.5-50.5$ \\
\hline K2 (D) & $44.18 \pm 2.34$ & $39.0-49.75$ \\
\hline Average K (D) & $44.35 \pm 2.21$ & $39.5-49.75$ \\
\hline \multicolumn{2}{|c|}{ AXL (mm) } & $21.36 \pm 0.50$ \\
\hline ACD (mm) & $3.38 \pm 0.52$ & $2.28-4.22$ \\
\hline Pre SE & $1.17 \pm 1.39$ & $0.0-4.37$ \\
\hline
\end{tabular}

Among study subjects, The mean Average K(D) was 44.35 \pm 2.21 , mean Axial length (mm) was 21.36 \pm 0.50 and the mean ACD (mm) was $3.38 \pm 0.52 \mathrm{~mm}$. preoperative mean spherical equivalent was $0.36 \pm 1.79$ (D).

Table-4: comparison of various parameters among 4 IOL calculating formula groups.

\begin{tabular}{|c|c|c|c|c|c|c|c|}
\hline Groups & Av K (D) & AXL $(\mathbf{m m})$ & $\begin{array}{c}\text { ACD } \\
(\mathbf{m m})\end{array}$ & SRK2 & HOLLA & HOFFQ & HAIGS \\
\hline Gr 1 & $44.78 \pm 2.47$ & $21.42 \pm 0.56$ & $3.51 \pm 0.54$ & $26.06 \pm 2.26$ & $25.70 \pm 2.26$ & $25.58 \pm .2 .11$ & $25.28 \pm 2.20$ \\
\hline Gr 2 & $45.23 \pm 2.67$ & $21.39 \pm 0.53$ & $3.47 \pm 0.58$ & $26.58 \pm 2.33$ & $26.22 \pm .22$ & $26.16 \pm 2.1$ & $25.86 \pm 2.29$ \\
\hline Gr 3 & $43.78 \pm 1.93$ & $21.36 \pm 0.36$ & $3.11 \pm 0.42$ & $25.82 \pm 1.12$ & $25.66 \pm 1.21$ & $25.46 \pm 1.17$ & $25.08 \pm 1.07$ \\
\hline Gr 4 & $44.18 \pm 2.45$ & $21.27 \pm 0.54$ & $3.44 \pm 0.46$ & $26.38 \pm 1.96$ & $26.02 \pm 2.00$ & $25.88 \pm 1.99$ & $25.70 \pm 1.89$ \\
\hline Anova F & 1.81 & 0.43 & 3.30 & 0.73 & 0.46 & 0.67 & 0.88 \\
\hline P value & 0.15 & 0.73 & $0.02 *$ & 0.54 & 0.71 & 0.57 & 0.45 \\
\hline
\end{tabular}

One Way ANOVA

$* \mathrm{P}<0.05$, Significant

$\mathrm{P}>0.05$, non-significant

On comparison of average K(D) and Axial length ( $\mathrm{mm}$ ) among the 4 groups using one Way ANOVA test, p- value of 1.15 and 0.73 was obtained, suggesting a statistically no significant correlation. But comparison of ACD among 4 groups shows p- value of 0.02 , suggesting statistically significant correlation. 
Original Research Article

\section{Discussion}

Cataract surgery is a keratorefractive procedure where the patient expects total visual rehabilitation in the form of improved vision, contrast sensitivity, and depth perception. The most important cause of a refractive surprise after IOL implantation is an incorrect IOL power calculation which is the single most important determinant of patient satisfaction after surgery. It depends on multiple variables such as AL, $\mathrm{K}, \mathrm{ACD}$, and ELP.

The commonly used third-generation formulae were Holladay 1, SRK-T (T for theoretical), and Hoffer Q which helped increase accuracy further. Each of these formulae estimates the position of the IOL within the eye based on the $\mathrm{K}$ and/or $\mathrm{AL}$, and the results are more accurate. Therefore, the use of older regression formulae has declined significantly in clinical practice. The quest for further accuracy led to the application of fourth generation formulae that use additional biometric parameters: Haigis formula requires $\mathrm{ACD}$ and ELP, Holladay 2 formula requires $\mathrm{ACD}, \mathrm{ELP}$ as well as white-to-white distance, lens thickness, preoperative refraction, and patient's age [5].

The evolution of IOL formulae has produced great accuracy but the goal of successfully reaching a given target refraction $100 \%$ of the time is still a difficult outcome to achieve as the ELP depends on different variables. The general principle behind every IOL formula is to calculate and predict the postoperative ELP. The first generation of IOL formulae considered the ELP to be a constant numeric value. Subsequent advanced theoretical and regression formulae began using more biometric data to calculate the ELP. The use of AL, corneal power, measured ACD, and white-to-white measurement further refined the accuracy of ELP prediction [6].

Holladay also found that eyes can have a shallow, normal, or deep anterior chamber in a short, average or long $\mathrm{AL}$ eyes. These variations can result in large differences in IOL calculations, and a small anterior chamber in a short eye one can expect the IOL power to be high. However, an equally short eye with deep anterior chamber will have a far larger IOL power and more uncertainty in IOL power calculation because the ELP will be farther from the cornea and closer to retina [7]. The conventional view is that shorter the ocular $\mathrm{AL}$, shorter should be the ACD. In the present study also as the mean AL decreased from $21.99 \mathrm{~mm}$ to $19.98 \mathrm{~mm}$ correspondingly the ACD also decreased from $4.22 \mathrm{~mm}$ to $2.28 \mathrm{~mm}$. A Chinese study by Chang John in 2012 found that there was a statistically significant positive correlation between AL and ACD in normal and long eyes but not in extremely long eyes [8]. Another American study in 2008 by Jivrajka $\mathrm{R}$ found a positive correlation between AL and $\mathrm{ACD}$ and an inverse correlation between AL and Lens thickness [9]. Although ACD should decreases with decreasing $\mathrm{AL}$, it was surprising to see significant variation in the ACD in eyeballs of nearly the same size [10]. This prompted to do a differential group analysis, and the ACD was examined in the eyes of $\mathrm{AL}<19,19-20,21-22 \mathrm{~mm}$. There was a wide variation in ACD and eyes with AL 20$21 \mathrm{~mm}$ mean ACD was 2.65 $\pm 0.24 \mathrm{~mm}$ with Avg k-value $43.71 \pm 2.55 \mathrm{~mm}$ with IOL power (SRK-T) ranging from 19.5D to $27.5 \mathrm{D}$. In eyes with AL 21-22 mm mean ACD ranged was $3.60 \pm 0.36 \mathrm{~mm}$ with $\mathrm{IOL}$ power ranging widely from $17 \mathrm{D}$ to $27.5 \mathrm{D}$.

Similarly, there was wide variation in ACD and consequently in the power of the IOL to be implanted in eyes with comparable AL. Within the group with similar $\mathrm{AL}$, there was no correlation between AL and ACD. This signifies either a difference in lens thickness or a difference in position of the Lens. Variable lens position would result in inevitable variability of ELP, which would influence the IOL power calculation in various fourth-generation formulae that incorporate ELP.

An extensive literature search was carried but could not find any study that tried to determine AL-Specific correlation with ACD. The present study was undertaken to determine if there was indeed any linear correlation between AL and $\mathrm{ACD}$. If $\mathrm{AL}$ and $\mathrm{ACD}$ are correlated only then can one assume that the position of the Lens is fixed and therefore ELP can be accurately calculated. If it was otherwise or the correlation was weak or nonlinear, then it is very difficult to determine postoperative ELP which is a critical determinant of IOL power in fourth-generation formulae.

The central thickness of the Lens and its position would have to be considered in estimating ELP without which fourth-generation formulae would be inherently unpredictable.

This study establishes that there is weak linear positive correlation between ACD and AL. Although fourth generation IOL Formulae are superior to all previous formulae since they still rely on ELP (a parameter that differs in the eyes of even same size), they are inherently prone to error.

It was observed that as overall mean AL decrease overall mean ACD also decreased but the differential correlation between different AL-based groups was very weak.

In all previous studies that was taken into account, it was found in the literature, the correlation between AL and ACD was determined, but there was no analysis for eyes with similar AL. 


\section{Original Research Article}

A potential limitation of the present study pertains to the fact that

1. Partial coherent interferometry method (IOL Master) was not used, which is considered as most accurate method in IOL calculation

2. Applanation A-scan method was used instead Immersion method, which could be a potential source of error in Axial length calculation.

\section{Conclusion}

As a general trend, decrease in mean ocular AL is accompanied with a decrease in ACD and it was also observed the same trend. However, the further differential analysis shows that within the eyes with near-equal $\mathrm{AL}$ there is wide variability in ACD. This may be due to either varying central lenticular thickness or due to the position of Lens in the anterior segment. The position of the Lens has a direct bearing on the ELP following IOL implantation.

Therefore, ELP based formulae (Haigis and Holladay 2) Have an inherent element of unpredictability and may result in postoperative refractive surprises. Further larger population-based studies need to be done in this regard, to assess the efficacy and importance of this study in eyes with axial length less than $22.00 \mathrm{~mm}$.

\section{What this study adds to existing knowledge?}

The present study throws a light on the difference in ACD within the eyes with near equal AL which may be due to either varying central lenticular thickness or due to the position of Lens in the anterior segment

\section{Author's contribution}

Dr. Savitha D P.: Concept, study design

Dr. Anusha N. Sangapur: Data analysis, manuscript preparation

Dr. Lakshmi B R.: Manuscript preparation

Funding: No funding sources

Conflict of interest: None declared

Ethical Approval: This study was approved by the Institutional Ethics Committee.

\section{References}

1. Survey of Blindness-India, NPCB-WHO Report. New Delhi: Ministry of Health \& Family Welfare, Government of India; 2015-2018.

2. World Health Organization. The World Health Report, Life in 21st Century - A Vision. Geneva: World Health Organization; 2018.

3. Williams HP. Sir Harold Ridley's vision. Brit J Ophthalmol. 2001;85(9):1022-1023. doi: http://dx.doi. org/ 10. 1136/bjo.85.9.1022.

4. David J. Sir Herold Ridley \& His Fight for Sight, He Changed the World so that we may Better. New Jersey; 2006. Available from: http://www. DR.Hills.com/iol/ haigis. [Last accessed on 2018 Aug 15].

5. Gavin EA, Hammond CJ. Intraocular lens power calculation in short eyes. Eye 2008;22(7):935-938. doi: $10.1038 /$ sj.eye. 6702774 .

6. Devgun U. Ocular surgery. US Edition. 2016;3:1327.

7. Hoffer KJ. Clinical results using the Holladay 2 intraocular lens power formula. J Cataract Refract Surg. 2000; 26(8):1233-1237.

8. Chang, John S. M.; Lau, Silvania Y. F. Correlation Between Axial Length and Anterior Chamber Depth in Normal Eyes, Long Eyes, and Extremely Long Eyes. Asia Pac J Ophthalmol. 2012;1(4):213-215. doi: 10.1097/APO. 0b013e31825f8cb6.

9. Jivrajka R, Shammas MC, Boenzi T, Swearingen M, Shammas HJ. Variability of axial length, anterior chamber depth, and lens thickness in the cataractous eye. J Cataract Refract Surg. 2008; 34(2):289-294. doi: 10.1016/j.jcrs. 2007. 10.015 .

10. Holladay JT. Refractive power calculations for intraocular lenses in the phakic eye. Am J Ophthalmol. 1993; 116(1): 63-66. doi: https://doi.org/10.1016/S00029394 (14)71745-3.

\section{How to cite this article?}

Savitha DP, Sangapur AN, Lakshmi BR. Correlation between ocular axial length, anterior chamber depth, Average K-values and a differential analysis in short Trop J Ophthalmol Otolaryngol.2019;4(8):452-456.doi:10.17511/jooo.2019.i08.01 